\title{
La Première Restauration face à « l'inexplicable Vendée » : la levée de boucliers des 3-5 mai 1814
}

\section{Aurélien Lignereux}

\section{(2) OpenEdition}

\section{Journals}

Édition électronique

URL : http://journals.openedition.org/abpo/2077

DOI : $10.4000 /$ abpo. 2077

ISBN : 978-2-7535-1841-4

ISSN : 2108-6443

Éditeur

Presses universitaires de Rennes

Édition imprimée

Date de publication : 30 décembre 2011

Pagination : 63-89

ISBN : 978-2-7535-1839-1

ISSN : 0399-0826

\section{Référence électronique}

Aurélien Lignereux, « La Première Restauration face à « l'inexplicable Vendée » : la levée de boucliers des 3-5 mai 1814 ", Annales de Bretagne et des Pays de l'Ouest [En ligne], 118-4 | 2011, mis en ligne le 30 décembre 2013, consulté le 20 avril 2019. URL : http://journals.openedition.org/abpo/2077 ; DOI : $10.4000 / a b p o .2077$ 


\title{
La Première Restauration face à "l'inexplicable Vendée " : la levée de boucliers des 3-5 mai 1814
}

\author{
Aurélien LiGNEREUX \\ Maître de conférences d'hsitoire contemporaine \\ Université d'Angers - CERHIO UMR 6258
}

Écrivant à son père le 4 mai 1814, dans une ville de Nantes sur le quivive à l'annonce d'une prise d'armes sur la rive gauche de la Loire, le préfet Prosper de Barante affecte d'en sourire : « Les campagnes sont en plein soulèvement. Contre qui? elles n'en savent rien; mais prendre un fusil est une manière d'exprimer sa pensée dans la Vendée ${ }^{1}$. " Ce mot d'esprit, qui assure à l'épisode quelques traces historiographiques ${ }^{2}$, vaut surtout pour son ambivalence : l'ironie envers les emportements des Vendéens n'en occulte ni la récurrence ni la gravité : vingt ans de luttes ont remplacé la faux par le fusil dans les représentations. C'est reconnaître la sensibilité politique exacerbée d'une région dont l'identité s'est forgée au feu de l'insurrection et de la répression. De fait, à 32 ans, Barante n'en est pas moins un fin connaisseur de la contrée qu'il administre depuis 1807, d'abord comme sous-préfet à Bressuire, puis en tant que préfet de la Vendée et enfin de la Loire-inférieure. Cet auditeur au conseil d'État incarne les élites promues sous Napoléon et ralliées sans peine à la Restauration. Barante a aussi ceci de remarquable, et finalement de représentatif, c'est que, tout en admirant les Vendéens de 1793 - les Mémoires de la marquise de La Rochejaquelein lui doivent beaucoup -, il méprise les revendications de la génération suivante.

Les révolutionnaires ont été confrontés à "l'inexplicable Vendée", le soulèvement de tout un peuple contre une révolution menée au nom du

1. Barante, Claude de (éd.), Souvenirs du baron de Barante, Paris, Calmann-Lévy, 1892, II, p. 63 .

2. BERTIER DE SAUvignY, Guillaume, La Restauration, Paris, Flammarion, 1990 [1963], p. 55. Notons cependant que la consciencieuse biographie de Barante par Antoine Denis ne mentionne pas l'événement (Amable-Guillaume-Prosper Brugière Baron de Barante (17821866). Homme politique, diplomate et historien, Paris, Champion, 2000, 1005 p. 
peuple. Vingt ans plus tard, les certitudes de la Restauration sont momentanément ébranlées par une incompréhension du même ordre : la fronde de la "fidèle Vendée " contre les autorités reconnues par le roi légitime. C'est annoncer la difficulté à appréhender l'événement par delà l'écran des interprétations des contemporains ${ }^{3}$, difficulté que redouble le déroulement des faits : cette prise d'armes du 3 au 5 mai 1814 n'a guère été émaillée de violences. C'est là l'une de ses spécificités mais aussi la cause de son oubli, non seulement parce que la mémoire des transitions de 1814-1815 s'est fixée sur des événements plus saillants, pour ne pas dire sanglants - la quatrième guerre de Vendée ou la Terreur blanche -, mais encore parce que la prise d'armes de mai 1814 n'a pas donné lieu à une procédure judiciaire, privant par conséquent l'historien de sources de choix.

Or cet épisode n'en est pas moins porteur d'enjeux propres à nourrir certaines tendances du renouveau de l'histoire du premier XIX ${ }^{\mathrm{e}}$ siècle, en particulier le regain d'attention prêté aux rumeurs ${ }^{4}$, les voies plurielles d'accès au politique en dépit des entreprises d'exclusion et en deçà des définitions établies ${ }^{5}$, ou les phases de transition ${ }^{6}$, dont celle méconnue de 1814 malgré la lumière crue qu'offre la vacance du pouvoir sur la vitalité des alternatives ${ }^{7}$. De fait, cet événement soulève la question d'une politisation blanche, restée invisible au sein de l'historiographie en raison d'un ostracisme qui n'a plus lieu d'être ${ }^{8}$. Ce mouvement invite enfin à tirer parti d'une figure du répertoire contestataire, à valeur générique - la levée de boucliers -, pour laquelle tout reste à faire, à commencer par l'enquête lexicologique ${ }^{9}$. En première approche, l'expression désigne la prise d'armes d'une partie significative d'une population, mobilisée de manière défensive pour manifester son mécontentement et intimider le pouvoir en place, occupant le terrain qui lui est familier pour faire face à ce qu'elle ressent comme une agression.

3. Pour un écho des débats, BiRÉ, Édouard (éd.), Mémoires du général d’Andigné, Paris, Plon, 1901, II, p. 190-191.

4. Ploux, François, De bouche à oreille : naissance et propagation des rumeurs dans la France du XIXe siècle, Paris, Aubier, 2003, 289 p.

5. ColAntonio, Laurent, FuReix, Emmanuel, JARRIGE, François (dir.), La souveraineté populaire. Expériences et normalisations en Europe (1800-1848), $\mathrm{n}^{\circ} 42,1^{\mathrm{er}}$ sem. 2011 de la Revue d'histoire du XIX ${ }^{e}$ siècle; OfFERLÉ, Michel, Ploux, François, LE GALL, Laurent (dir.), La politique informelle en France et en Europe (XIXe-XXI siècle), Rennes, PUR, à paraître.

6. Rappelons ici le dossier "Gendarmerie et transition(s) politique(s) au XIX siècle ", Annales de Bretagne et des Pays de l'Ouest, t. 114, juin 2007.

7. BERCÉ, Yves-Marie, "L'ombre d'une politique populaire au printemps 1814 », La fin de l'Europe napoléonienne. 1814 : la vacance du pouvoir, Paris, Veyrier, 1990, p. 330-331.

8. Un rattrapage est en cours. À titre d'illustration pour le Midi blanc: TRIOMPHE, Pierre, "Au nom de Dieu, du roi et de tous les miens. Imaginaire, sociabilité et expressions politiques des classes populaires royalistes dans la France méridionale (1800-1851) ", Annales du Midi, t. 123, n 274, avril-juin 2011, p. 195-212.

9 . Pour s'en tenir aux repères des dictionnaires du $\mathrm{xIX}^{\mathrm{e}}$ siècle, l'expression dérive de l'usage qu'avaient les légionnaires de lever leur bouclier en présence de leur général, en geste de désobéissance. Elle désigne ensuite métaphoriquement une opposition armée; les occurrences citées font la part belle au contexte vendéen. Le Dictionnaire de l'Académie française (1835) précise que cette démonstration se fait avec éclat et sans succès. 


\section{"Les deux partis extrêmes se sont épouvantés l'un l'autre : le plus fort a pris les armes ${ }^{10}$ "}

C'est dire si un tel sursaut, fruit d'une maturation qui se révèle au choc d'une provocation, ne peut se comprendre sans la configuration locale d'avril 1814, bien que cette mise en contexte soit tributaire d'un effet de source. Soucieux d'expliquer des troubles qui les ont surpris, les administrateurs de la Restauration ont collecté et suscité des informations, qui, à leur lueur, pouvaient apparaître comme les signes précurseurs d'une explosion inéluctable. Il convient également de faire ressortir le décalage entre l'événementiel parisien (déchéance de Napoléon par le Sénat et appel à Louis XVIII, du 3 au 6 avril) et la réception de ces nouvelles, retardées en Vendée jusqu'au 10 avril par le préfet, qui n'arbore le drapeau blanc que le surlendemain. Et encore faut-il tenir compte du délai d'accréditation par les habitants et, en sens inverse, de la déformation préfectorale des avis sur l'Ouest tels qu'ils parviennent à Paris.

Cette chronologie parallèle accentue les problèmes en suspens. Il y a d'abord l'agitation d'une contrée restée en situation pré-insurrectionnelle. Dès 1813, des insoumis opéraient des coups de main, mais les chefs freinaient des initiatives jugées prématurées tandis que le déploiement de la gendarmerie d'Espagne assurait la présence encore dissuasive de l'État napoléonien. La quatrième guerre de Vendée n'a pas eu lieu, du moins pas en 1814. Tout en faisant avorter un soulèvement prévu le 11 avril, l'effondrement soudain du régime à Paris laisse en place les administrations départementales. Rallié au roi, son personnel continue de fustiger les bandes qui ne désarment pas. L'inquiétude indignée des autorités est accrue par leur conviction que ces insoumis sont soudoyés au service d'une politique de réaction. Dès le 25 avril, Bernard-Laducquerie, sous-préfet à Fontenay, réactive ainsi la thèse du complot nobiliaire, tout en laissant entrevoir que ces recrues entendent profiter du rapport de forces pour obtenir la fin des impôts.

De fait, en réaction aux exigences de l'Empire aux abois, l'exaspération antifiscale est alors générale, mais elle prend une dimension particulière dans l'Ouest, en raison de la promesse de cinq ans d'exemption accordée par les chefs pour galvaniser les futurs insurgés. La répugnance à verser les contributions est exacerbée par le maintien des percepteurs napoléoniens, ce qui fait craindre que l'argent des royalistes ne serve de trésor de guerre contre le roi. Convaincus que l'épreuve de force perdure, les Vendéens s'irritent de l'avantage dont disposent leurs adversaires restés en fonction. La gendarmerie fait figure de force de frappe jacobine, ce qui pousse les royalistes à ne compter que sur leurs propres moyens en cas d'attaque. Il est vrai que la spirale des violences depuis 1793 a remis l'auto-défense à l'honneur. Le discrédit de l'administration en 1814 favorise aussi l'affirmation de contre-pouvoirs, s'adressant au monarque par des députations. S'apprêtant à

10. Le commissaire du roi Gilbert des Voisins au commissaire à l'Intérieur, 16 mai 1814, Arch. nat., $F^{7} 7029$. 
gagner la capitale, Constant de Suzannet s'établit ainsi en censeur de Basset de Châteaubourg, impopulaire préfet de la Vendée pour y avoir été nommé en mars 1813 comme exécuteur des levées d'hommes répétées.

Or cette absence momentanée des grands chefs, réunis à Paris ou à Nantes, augmente le désarroi des royalistes. Chaque camp scrute avec anxiété les menées supposées de l'autre, dans la peur d'être pris au dépourvu. La circulation de fausses nouvelles, telles que des listes de proscription, alimente l'escalade, mécanique des peurs et des rancœurs mutuelles dont rend bien compte cette missive à un chef royaliste :

"Les gendarmes arrivent tous les jours en armes à Montaigu, on ne sait ni pourquoi ni par quel ordre, vous savez que cette espèce n'aime pas la cocarde blanche. Ils insultent ceux qui la portent. [...] Leur cri de ralliement est mourir pour mourir, il faut mourir les armes à la main, et détruire la race des prêtres et des nobles. [...] Il faut craindre aujourd'hui le fer et la flamme de ces cannibales révoltés et armés ${ }^{11}$."

Quant aux patriotes, dont l'infériorité numérique attise le sentiment de vulnérabilité, ils vivent dans la crainte d'être égorgés, les biens nationaux constituant le mobile du crime à venir comme s'en persuade Mercier du Rocher, un ancien administrateur de Fontenay ${ }^{12}$. Le contraste est vif avec le peu de crédit d'une parole officielle dévaluée, qui ne répond que par le mépris à l'angoisse que révèlent les rumeurs, à l'instar du préfet Busche raillant, par exemple, la fable d'un retour de Bonaparte à la tête d'une armée turque $^{13}$. La diffusion de cet imprimé coïncide avec celle du bruit qui met le feu aux poudres...

Toute proportion gardée, le phénomène s'apparente à la Grande Peur. Dans un climat d'anxiété quant au devenir d'une victoire qui paraît fragile tant elle est menacée, on annonce les ravages de bandes armées. Les villageois s'arment alors au son du tocsin, sillonnent le pays, accréditant de ce fait le danger auprès des communes voisines; faute de trouver l'ennemi redouté, certains groupes se retournent contre leurs adversaires locaux. Busche joue d'ailleurs de la référence pour mettre en garde contre l'amorce d'une révolution : "Comme au 14 juillet 1789, en un jour toute la population est sous les armes ${ }^{14}$. "L'analogie est d'abord formelle. C'est aisni que le 5 mai, 6000 hommes armés convergent à Nueil-sous-les-Aubiers, là même où s'étaient réunis 4000 à 5000 paysans vingt-cinq ans plus tôt. En écho aux bruits agitant le Poitou de $1789^{15}$, on annonce en 1814 l'irruption de Polonais, phantasme que motive cette fois l'expérience napoléonienne. Mais la résonance est plus profonde : est de nouveau à l'œuvre le savoir

11. Au colonel Duchaffaud, 30 avril 1814, Arch. nat., $\mathrm{F}^{7} 7029$.

12. GÉRARD, Alain (dir.), Napoléon et les Vendéens. Journal inédit de Mercier du Rocher (1799-1816), La Roche-sur-Yon, Centre vendéen de recherches historiques, 2004, p. 270.

13. Le préfet des Deux-Sèvres aux fonctionnaires du département, 3 mai 1814, Arch. nat., $\mathrm{F}^{7} 9226$.

14. Le préfet des Deux-Sèvres au directeur général de la police, 28 mai 1814, ibid.

15. LEFEBVRE, Georges, La Grande peur de 1789, Paris, A. Colin, 1988, p. 176 et 188. 
traditionnel de communautés perméables aux rumeurs, méfiantes à l'égard de l'étranger, sur la défensive face aux errants ${ }^{16}$. Cette prédisposition à l'alarme, en son sens originel, pèse d'autant plus qu'il ne faut pas compter sur le secours des villes enkystées dans le pays.

Les analyses de Marc Bloch, érigeant la fausse nouvelle en miroir de représentations collectives préexistantes nous ont habitué à sonder le discours rumoral ${ }^{17}$. À sa source, le bruit incrimine les ennemis intimes des Vendéens - on parle d'une colonne de mille à deux mille gendarmes et jacobins, commandée par le général Travot, vainqueur de Charette et acquéreur de biens nationaux, sortie de Montaigu et mettant tout à feu et à sang sur son passage. Très vite cependant, ce sont les suppôts de Napoléon qui défilent au fil des versions : garde impériale, lanciers polonais, gendarmes d'Espagne. En quête d'une explication raisonnable, les autorités penchent d'abord pour des douaniers licenciés, des déserteurs, des prisonniers de guerre en maraude, ou des "indépendants" - soldats rebelles à la monarchie restaurée et vivant sur le pays. La liste des victimes est également éloquente. Les bruits initiaux prolongent les alarmes d'avril : on parle de nobles et de prêtres assassinés, ce qui prouverait la manipulation selon certains, à l'exemple du maire de Saint-Révérend notant que les propagateurs débitent à la lettre le même texte ${ }^{18}$. Ces avis enflent cependant; il devient question de massacres et le chiffre de 200 femmes égorgées se répète dans les Mauges en une évidente résurgence du traumatisme des colonnes infernales de 1794.

Née au sein d'un terreau prédisposé, la rumeur entraîne une terreur panique - l'image de la vague est alors de mise -, qui ne saurait être réduite au seul registre de la crédulité paysanne, comme le prouve l'émoi de jeunes Angevins de bonnes familles " qui avaient la tête très exaltée et voulaient partir sans savoir où ils allaient, pour aller se battre, sans savoir contre qui ", que le préfet Hély d'Oissel juge prudent de canaliser en partant à leur tête ${ }^{19}$. La propagation tient à l'effet d'entraînement suscité par le jeu convergent du tocsin, des attroupements, de la transmission des nouvelles dans les foires, comme celle de Coëx, de la fuite de femmes et d'enfants hâtant celle de voyageurs et de patriotes inquiets des représailles, des menées de certains chefs. Si la rumeur se relaie rapidement, elle est vouée à retomber : les marches paysannes finissent par la démentir. Bref, la dynamique rumorale est celle d'une fuite en avant, mais destinée à se résorber au fur et à mesure. C'était sans compter les efforts de structuration.

16. WAHNICH, Sophie, L'Impossible citoyen. L'étranger dans le discours de la Révolution française, Paris, A. Michel, 1997, p. 103.

17. BLOCH, Marc, "Réflexions d'un historien sur les fausses nouvelles de la guerre ", dans : Écrits de guerre, 1914-1918, BLoch, Étienne (dir.), Paris, A. Colin, 1997 [1921], p. 181.

18. Le maire de Saint-Révérend au préfet de la Vendée, 5 mai 1814, Arch. nat., $\mathrm{F}^{7} 9099$.

19. Le préfet de Maine-et-Loire au commissaire à la Police générale, 4 mai 1814, Arch. nat., $F^{7} 9111$. 


\section{"Que le premier cerveau brûlé ne soit plus le maître de faire sonner le tocsin dans 300 communes et d'armer 50000 hommes ${ }^{20}$ "}

Des notables se sont posés en recours naturels des populations, rôle qu'avalise souvent leur fonction de maire. Du reste, une telle responsabilité venait d'être rappelée, le 15 avril, par une circulaire du commissaire du gouvernement chargé de l'Intérieur, enjoignant aux maires d'organiser la garde nationale sédentaire pour préserver leur commune " du pillage et des excès que pourraient commettre des détachements isolés " et des "maraudeurs étrangers". On conçoit que ce genre de consignes ait pu à la fois accréditer l'anxiété diffuse et légitimer l'appel aux armes, d'autant plus que l'uniforme de ces gardes "n'est pas de rigueur ${ }^{21}$ ". Les préfets admettent en tout cas la tutelle salutaire des maires propriétaires pour contenir les débordements de paysans à la fois effrayés et furieux. Non sans ambiguïté : certes, l'absence des grands chefs fait que « les paysans n'ont ni conseils, ni influence qui puisse les ramener à la raison ", mais, trahissant sa crainte d'un encadrement, Barante en conclut que l'alerte ne durera pas $^{22}$. Hély d'Oissel loue la conduite de M. de Chanzeaux, ancien seigneur de cette paroisse, qui s'est porté à la tête de 150 paysans jusqu'à Chemillé avant de les ramener chez eux, ayant constaté la fausseté des bruits. En revanche, venu reprendre la situation en main, le 5 mai à Beaupréau, le préfet blâme l'activisme irresponsable de MM. de La Béraudière et de La Sorinière, vu " que c'était une chose très fâcheuse que d'agiter et d'armer ainsi le peuple, qu'on était pas toujours sûr de le faire rentrer dans le devoir ${ }^{23}$ ".

En fait, ces chefs ont su encadrer un mouvement qui les rendait maîtres du pays, en lieu et place de l'administration. Au-delà des levées communales, ils travaillent à une mobilisation régionale. Dans les Mauges, le sous-préfet Barré doit s'incliner face à MM. de Lhullier, Barbier du Doré et Armaillé, entraînant la population aux armes, sous le titre de commissaires du roi, malgré la révocation de leur pouvoir. On signale çà et là des domestiques à chapeau rond et cocarde blanche, remettant à des notables les rôles de levée. Il en ressort que la prise d'armes a changé de nature au cours de sa propagation. À sa naissance, au sud de Nantes, il s'agit d'une réaction improvisée d'auto-défense pour parer un danger imminent, bien que le poids d'un chef subalterne comme Le Couvreur soit évident. Au fil des heures, la mobilisation se transforme, s'opérant par le haut dans les

20. Le sous-préfet de Beaupréau au préfet de Maine-et-Loire, 5 mai 1814, Arch. nat., $\mathrm{F}^{7} 9111$.

21. Le préfet de Maine-et-Loire aux sous-préfets et maires du département, 15 avril 1814, Arch. dép du Maine-et-Loire, 4 R 62.

22. Le préfet de la Loire-inférieure au commissaire à la Police générale, 3 mai 1814, Arch. nat., $\mathrm{F}^{7} 9250$.

23. Le préfet de Maine-et-Loire au commissaire à la Police générale, 8 mai 1814, Arch. nat., $\mathrm{F}^{7} 9111$. 
Mauges, se systématisant dans les communes de Vendée occidentale, où des estafettes ont précédé la rumeur.

Au total, la prise d'armes a touché quatre départements, de la Sèvre à la Loire, en un triangle Nantes-Saumur-Parthenay, terrain réceptif et réactif s'il en est. Plusieurs foyers ont été désignés : d'une part, les villes d'où serait partie la colonne incendiaire (Montaigu ou Nantes); d'autre part, les communes prétendument dévastées (Vallet, Clisson ou Vezins). Sous le coup de l'émotion, le sous-préfet de Beaupréau parle de 50000 hommes en armes; le 7 mai, le préfet des Deux-Sèvres en évoque 60000. Le commissaire Ruty réduira de moitié ce décompte.

La panique est née le 3 mai, vers 6-7 heures du matin, dans l'arrondissement de Nantes et autour de Montaigu dans la Vendée voisine. La nuit suivante, elle atteint le Maine-et-Loire, où le tocsin retentit de dix heures du soir à trois heures du matin dans l'arrondissement de Beaupréau et à l'ouest de celui de Saumur. Le mouvement se structure le 4 : en Vendée, des hommes sont enrôlés autour de Saint-Gilles; Le Couvreur entre dans Montaigu avec 800 hommes tandis que Beaupréau est occupée par les villageois des Mauges. Dans la nuit du 4, le tocsin alerte le Bocage des DeuxSèvres. Le reflux, déjà sensible la veille dans la Loire-inférieure, gagne le Maine-et-Loire. Le 5, les rassemblements au nord-ouest des Deux-Sèvres, se disloquent dans la matinée. Quelques bastions demeurent en Vendée : des attroupés restent en armes jusqu'au 7 chez M. de La Bastière, pérennisant l'inquiétude aux Sables, et c'est seulement le 8 que Le Couvreur disperse ses forces centrées sur Palluau-Legé.

La prise d'armes révèle ainsi le poids des chefs subalternes, élus capitaines par leurs paroisses. Une telle latitude de mobilisation choque les autorités. "Il importe que le droit d'agir soit réservé seulement aux personnes revêtues d'un caractère public ", s'emporte le préfet Châteaubourg ${ }^{24}$. À cette dépossession s'ajoute l'indignation face à l'insignifiance sociale des chefs locaux. Louis Le Couvreur, ancien soldat de Charette, doit son influence au vivier de recrutement des environs de Legé. Ce tisserand de métier et marchand de vin en détail est considéré avec égard par ses supérieurs, supérieurs dont l'entremise a été sollicitée par les préfets. C'est là un double aveu : celui de l'impuissance des autorités; celui d'une dépendance des grands chefs envers ces meneurs d'hommes, en un écho atténué des tensions nées de la dualité de l'encadrement au cours des guerres de l'Ouest. Encore Le Couvreur jouit-il d'une certaine respectabilité. Le discours préfectoral se fixe volontiers sur des subordonnés au passé trouble, tels Chardonnet et Thomazeau, mis à prix dès décembre 1813, voire sur d'inquiétants hommes de main (Fillabre, Soret ou Gazeau).

C'est poser la question des violences dans le sillage d'une telle prise d'armes, et, dès lors, s'interroger sur les cibles dérivées une fois dissipée

24. Le préfet de la Vendée au commissaire à la Police générale, 7 mai 1814, Arch. nat., $F^{7} 9099$. 
l'ombre d'un ennemi imaginaire. Le sous-préfet de Beaupréau évoque certes des individus assommés à Saint-Macaire ou à Gesté et dénonce « la licence, l'ivresse et la terreur " en un " jour du deuil public et de la vengeance 25 ", mais, exceptées ces déclarations dictées par le ressentiment, c'est le soulagement qui prime tant il y avait lieu de craindre des règlements de compte contre les patauds, anciens révolutionnaires, fonctionnaires et propriétaires de biens nationaux. Seule une poignée d'entre eux a été molestée. Beaucoup ont pris les devants : Renaud, maire de Saint-Gilles et conseiller général, se réfugie aux Sables; les acquéreurs du nord de la Vendée gagnent Nantes plutôt que Bourbon-Vendée où ils ne s'estiment pas à l'abri. Le 8 mai, le préfet Châteaubourg, qui redoute la fixation des antagonismes, exhorte ces réfugiés à rentrer chez eux. Instruit que leur présence à Nantes relance les rumeurs - ces anciens révolutionnaires y recruteraient des troupes et aveugleraient les autorités -, Barante ira jusqu'à les accompagner à Legé, pour présider en un rituel de paix à une sorte de pacte de sécurité nouée entre les réfugiés et les chefs de Roche-Servière, après les avoir convaincus successivement " ce qui est à peu près vrai, qu'ils s'étaient par mal entendu inspirés une terreur réciproque ${ }^{26}$ ".

Les brigades les plus exposées ont reçu l'ordre de se replier. En poste à Chemillé, un officier confiera qu' «il avait passé la journée dans de cruelles inquiétudes, craignant à chaque instant de voir ses gendarmes attaqués par les paysans qui tenaient les plus mauvais propos ${ }^{27}$ ". La gendarmerie n'a pas été attaquée ni même désarmée, mais son autorité s'est effondrée, laissant les administrateurs sans moyen d'action, pour ne pas dire sans protection. Même la garde nationale des chefs-lieux ne leur a pas offert son secours, parce que ses membres ne tenaient pas à se compromettre face à des hommes entrant, comme à Bressuire, aux cris de "Vive le Roi ". Il s'ensuit que les maisons du sous-préfet, du lieutenant de gendarmerie ou du procureur ont été violées. À Beaupréau, le sous-préfet Barré a dû se cacher dans une haie pour échapper à des hommes décidés à l'abattre, et qui, faute de le trouver, ont pillé son hôtel, sac qu'il impute à des déserteurs excédés par ses poursuites passées. Le maire de Montaigu, acquéreur de biens nationaux, a été sauvé par un curé. Il devait son impopularité à sa nomination au cours de l'hiver 1814, en remplacement du précédent maire qui opposait, lui, une force d'inertie aux exigences du pouvoir.

La prise d'armes s'est donc tournée contre les villes, dans le prolongement de la Révolution. Barré y voit l'influence des chefs, "qui montent la tête des paysans ", en leur désignant Montaigu, Cholet et Chalonnes

25. Le sous-préfet de Beaupréau au commissaire à la Police générale, 5 mai 1814, Arch. nat., $\mathrm{F}^{7} 9111$.

26. Le préfet de la Loire-inférieure au commissaire à l'Intérieur, 6 et 8 mai 1814, Arch. nat., F 1cIII L-I, 11.

27. Le préfet de Maine-et-Loire au commissaire à la Police générale, 8 mai 1814, Arch. nat., $F^{7} 9111$. 
comme hostiles au roi, et appelant dès lors à " tomber en force sur ces villes et les écraser ${ }^{28}$ ». L'occupation de Montaigu ou de Bressuire a donné lieu à des scènes inscrites dans le répertoire séculaire des marches paysannes : saisie des armes chez les fonctionnaires, visite des maisons bourgeoises pour en contraindre les propriétaires à régaler la troupe. Les préfectures, pourvues de troupes, n'ont pas été investies. Les Nantais, hantés par les précédents de juin 1793 et d'octobre 1799, ont néanmoins craint les représailles ou le pillage, obligeant les autorités à prendre des mesures de vigilance auxquelles elles répugnaient de peur d'alimenter les soupçons des ruraux.

\section{"Une fausse démarche aurait pu amener la guerre civile ${ }^{29}$ "}

La prudence a dicté la conduite des préfets, pariant sur la retombée de la panique et, de toutes façons, privés de moyens d'action. Châteaubourg se targue d'avoir évité le cycle provocation-répression-radicalisation, assurant que la manière forte aurait poussé à la révolte " 10000 autres ${ }^{30}$ ". La gendarmerie fait profil bas. Ses officiers sont bien conscients qu'une expédition serait interprétée comme une persécution des royalistes. Seul un parfait pataud tel que Gillardeau réclame l'appui de la troupe - « la force et la crainte peuvent seules désormais en imposer ${ }^{31}$ "-, mais le sous-préfet de Saumur est blâmé pour avoir envoyé des garnisaires dans quelques villages soulevés ${ }^{32}$. Pour apaiser les esprits, des militaires sont écartés. Cette défiance entretient leur malaise, comme en témoigne un épisode qui serait burlesque s'il ne s'était pas tragiquement conclu. Excédé par les égards accordés aux députés envoyés à Bourbon-Vendée par les attroupés de Roche-Servière, le major du $26^{\mathrm{e}}$ de ligne, qui voulait les arrêter comme séditieux, se brûle la cervelle, persuadé d'une conjuration contre l'armée. C'est la seule victime de ces événements.

Mai 1814 offre ainsi le rare exemple d'une prise d'armes qui n'a pas été réprimée. Le gouvernement s'en est tenu à la nomination d'un commissaire extraordinaire pour les $12^{\mathrm{e}}$ et $22^{\mathrm{e}}$ divisions militaires - le général Ruty, officier de l'artillerie napoléonienne - flanqué par les chefs vendéens historiques (Andigné, Autichamp, La Rochejaquelein, Suzannet, Sapineau), maîtres du jeu politique local, notamment La Rochejaquelein accusé de faire, au propre comme au figuré, cavalier seul. Cet amalgame est caractéristique de " l'anarchie paternelle " d'un régime soucieux de réunir les

28. Le sous-préfet de Beaupréau au commissaire à la Police générale, 5 mai 1814, Arch. nat., $\mathrm{F}^{7} 9111$.

29. Le préfet de la Vendée au directeur général de la Police, 4 juin 1814, Arch. nat., $\mathrm{F}^{7} 9250$.

30. Le préfet de la Vendée au commissaire à l'Intérieur, 5 mai 1814, Arch. nat., F 7029 .

31. Le procureur de Bourbon-Vendée au procureur général de Poitiers, 7 mai 1814, Arch. nat., $\mathrm{F}^{7} 9099$.

32. Le commissaire extraordinaire Ruty au préfet de Maine-et-Loire, 14 mai 1814, Arch. dép. du Maine-et-Loire, M 623. 
deux France et d'abord de mieux connaître le pays ${ }^{33}$ : ces commissaires se superposent en effet à ceux déjà nommés pour l'ensemble du royaume, le 22 avril, dans le cadre d' " enquêtes de réappropriation ${ }^{34}$ ". Cette première vague a échoué, le tandem Gilbert des Voisins et le vicomte d'Osmond n'ayant pas pu, mais en avaient-ils le temps, désamorcer les tensions. Le premier, placé à la tête de la garde nationale de Paris peu avant la chute de Napoléon, était d'emblée discrédité pour s'être opposé, disait-on, à la cocarde blanche jusqu'à l'entrée des alliés.

L'œuvre des commissaires s'est bornée à une tournée de pacification. Au fil des étapes, les mêmes séquences se répètent : accueil enthousiaste, rencontre avec les maires, juges de paix et curés, audiences aux habitants, discours d'ordre et de paix, exhortation à s'acquitter des impôts, déception des populations manifeste à l'heure du départ et qui affleure sous la satisfaction officielle. L'enquête administrative sur les causes voire sur les responsables de la prise d'armes rejette quant à elle la thèse défendue par le préfet Busche, celle d'une levée de boucliers comme levier de pouvoir pour des chefs vendéens frustrés du mérite d'avoir renversé le pouvoir napoléonien. Ils auraient alors constitué une armée " lorsque le Roi n'en avait pas besoin ", dans le but d'opérer « un soulèvement pour s'arroger le mérite de le réduire ". Reste l'obstacle incarné par les fonctionnaires qui "ne pouvaient être dupes de cette jonglerie ", et qu'il faut donc écarter, ce qui explique l'animosité que Busche suscite ${ }^{35}$.

Les commissaires préfèrent s'en tenir à l'idée d'un mouvement spontané, lié à la force d'inertie d'une insurrection organisée de longue date, et vouée à éclater comme un ressort trop longtemps comprimé. Le gouvernement s'y rallie avec soulagement : " Je pense ainsi que vous qu'il est inutile de s'appliquer à découvrir la cause des agitations [...]. À défaut de notions positives, je trouve que vos conjectures expliquent les faits de la manière la plus vraisemblable ${ }^{36}$." Le gouvernement, qui ne souhaitait pas d'épuration massive propre à compromettre le ralliement des patriotes, doit néanmoins révoquer les préfets de Maine-et-Loire, des Deux-Sèvres et de la Vendée, les sous-préfets de Beaupréau, de Bressuire, des Sables et de Saumur, le maire de Montaigu, des percepteurs et nombre de gendarmes.

33. Yvert, Benoît, WARESQuiel, Emmanuel de, Histoire de la Restauration (1814-1830), Paris, Perrin, 1996, p. 67-102.

34. BouRGUINAT, Nicolas, « Les commissaires extraordinaires de 1814-1815 : une mission de reconnaissance du territoire national ", in Nicolas BouRGUINAT et Sylvain VENAYRE (dir.), Voyager en Europe de Humboldt à Stendhal. Contraintes nationales et tentations cosmopolites, 1790-1840, Paris, Nouveau Monde, 2007, p. 325-344; KARILA-COHEN, Pierre, L'État des esprits. L'invention de l'enquête politique en France (1814-1848), Rennes, Presses universitaires de Rennes, 2008, p. 115-119.

35. Le préfet des Deux-Sèvres au directeur général de la Police, 28 mai 1814, Arch. nat., $\mathrm{F}^{7} 9226$.

36. Le ministre de l'Intérieur au commissaire d'Osmond, 4 juin 1814, Arch. nat., F7 7030. 


\section{" Ils ne seront satisfaits que lorsque tout sera remis sur le pied de $1789^{37}$ "}

Insurrection à contretemps, tel est donc le mot de la fin assigné à un mouvement ayant réussi à emporter les restes du régime détesté. Or cette prise d'armes est surtout à contre-courant. Elle a toute sa place au sein des crises de transition, propices à l'irruption d'alternatives radicales, disqualifiées tant par les dirigeants de veille que par ceux du lendemain. Barante évoquait un " état d'anxiété ", où tout semble possible, mais qui " cessera dès que le roi aura prononcé sur la constitution et aura pris en main les rênes du gouvernement ${ }^{38}$ ". Il était déjà acquis que le nouveau régime parierait sur une politique de réconciliation pour jouir d'une double continuité : celle séculaire de la monarchie des Bourbons; celle de l'État réinventé par vingt-cinq ans d'expériences. Dessein apaisant à la merci des vengeances, aussi est-ce le pardon qui scande le discours officiel et que relaient les préfets parce qu'ils en sont les pivots et les bénéficiaires.

La prise d'armes s'apparente dès lors à une violation de la volonté du roi en plus d'une transgression des lois. Il est vrai que la Déclaration de SaintOuen, du 2 mai, qui confirme la vente irrévocable des biens nationaux, était encore inconnue en Vendée au début des troubles. Sa diffusion contribue au retour au calme vu qu'elle rassure les patriotes dont la nervosité entretenait l'anxiété des royalistes et qu'elle dissuade ces derniers de braver ouvertement la parole royale. Indépendamment de son contenu, la Déclaration clôt l'interrègne durant lequel d'autres personnes que les fonctionnaires avaient pu s'exprimer, tel que M. de La Sorinière, qui, le 5 mai, bravait le préfet Hély d'Oissel, en déclarant " qu'ils verraient ce qu'ils auraient à faire quand le Roi aurait parlé lui-même ${ }^{39}$ ". Ceci étant chose faite, pareille attitude relève de la rébellion. S'obstiner à peser les armes à la main pour une autre issue politique serait une aberration, celle d'un " mouvement révolutionnaire de la part des hommes qui se sont toujours montrés [1]es partisans les plus dévoués [...] de l'auguste famille des Bourbons ${ }^{40}$ ". Il est sûr en tout cas que le mouvement de mai 1814 est contre-révolutionnaire, même en tenant compte du filtre des autorités qui se plaisent à souligner la nature rétrograde des revendications :

«Ils veulent que les écus de 6 livres soient pris pour leur valeur ancienne, que le mode de perception soit changé et rétabli comme autrefois au moyen de collecteurs; ils demandent que le droit sur le sel soit aboli, le pays ayant été rédimé; ils ne veulent pas entendre parler de droits réunis, d'octrois, etc. Ils ajoutent que si les biens de l'Église étaient rendus, on n'aurait plus

37. Le commissaire du roi Gilbert des Voisins au commissaire à l'Intérieur, 7 mai 1814, Arch. nat., $\mathrm{F}^{7} 7029$.

38. Le préfet de la Loire-inférieure au commissaire à la Police générale, 3 mai 1814, Arch. nat., $\mathrm{F}^{7} 9250$.

39. Le préfet de Maine-et-Loire au commissaire à la Police générale, 8 mai 1814, Arch. nat., $\mathrm{F}^{7} 9111$.

40. Le directeur général de la Police à M. de La Rochejaquelein, 18 mai 1814, Arch. nat., $\mathrm{F}^{7} 9099$. 
à payer pour les prêtres et les hôpitaux [...] d'après leurs idées qu'on leur avait probablement inculquées, le retour du roi devait être l'annonce du renversement de toutes les lois rendues dans le temps de la Révolution et de toutes les autorités quelconques, et le rétablissement pur et simple de l'ancien ordre des choses ${ }^{41}$."

La référence à l'insurrection héroïque de 1793, sous les traits exclusifs d'un combat pour Dieu et le Roi, sert à discréditer la réplique sans risque ni raison de 1814. C'est faire bon marché du faisceau des causes de mars 1793, dont l'écho perdure vingt ans plus tard, comme la méfiance envers les autorités citadines, le contentieux au sujet des biens nationaux, les séquelles des serments - on incrimine la Petite Église - et même le thème de la défense des libertés, mis à l'honneur dans les années 1980 par l'aggiornamento de l'historiographie blanche dans un sens antitotalitaire. Il est vrai que M. de La Sorinière, dont la famille a payé un lourd tribut à la guillotine début 1794 si bien que les dames de La Sorinière seront promues au rang de martyres emblématiques de la Terreur régionale, invoque fièrement la légitimité de la résistance contre la tyrannie - " nous voulons faire voir que nous sommes en mesure de Résister à l'oppression ${ }^{42}$ " -, face au préfet de Maine-et-Loire, qui y décèle un relent d'un autre 1793, celui de la Constitution montagnarde. Il serait sans doute plus juste d'y voir une réminiscence du « devoir de révolte " face au renforcement du pouvoir central ${ }^{43}$.

C'est déjà faire lit de la sédition. Le discours officiel s'en tient au raisonnement suivant : le roi ayant regagné son trône, les Vendéens n'ont plus qu'à rentrer chez eux. Les commissaires reprennent cette antienne et appellent au nom d'une même fidélité les combattants d'hier à se convertir en contribuables diligents. L'heure est à la démobilisation. Au fond, mai 1814 est un exemple d'échec d'une sortie de guerre. Gilbert des Voisins souligne ainsi l'altération du lien civique en Vendée et la difficulté à y réintroduire l'État : "Vingt années de guerre, l'habitude d'une résistance continuelle aux différents gouvernements qui se sont succédés pendant ce laps de temps ont fait germer ici des idées d'indépendance et établi l'influence d'un certain nombre de personnes, nuisibles à l'autorité même du roi et à la considération due à ses agents ${ }^{44}$. " La guerre a ruiné le contrat social. Pour refermer cette parenthèse, qui dépasse donc l'intervalle de vacance du pouvoir, une entreprise de normalisation est esquissée.

Elle passe par le désarmement. Vu le rapport de forces, les autorités renoncent à la livraison des armes et se satisferaient de la fin de leur port ostensible au nom de la paix civile : "Les habitants de la Vendée et de la

41. Le préfet des Deux-Sèvres au commissaire à l'Intérieur, 17 mai 1814, Arch. nat., $\mathrm{F}^{7} 9099$.

42. Le préfet Hély d'Oissel au commissaire à la Police générale, 8 mai 1814, Arch. nat., $\mathrm{F}^{7} 9111$.

43. JouAnna, Arlette, Le Devoir de révolte : la noblesse française et la gestation de l'État moderne (1559-1661), Paris, Fayard, 1989, 504 p.

44. Le commissaire du roi Gilbert des Voisins au commissaire à l'Intérieur, 16 mai 1814, Arch. nat., $\mathrm{F}^{7} 7029$. 
Loire-inférieure du même côté, ont conservé leur attitude guerrière, sans que le motif d'une telle levée de boucliers soit encore connu. [...] Il est instant que le gouvernement se prononce contre un armement qui répand l'épouvante ${ }^{45}$. " Le monopole étatique est à restaurer. Au cœur du soulèvement, les autorités s'étaient résignées à livrer « le maintien de la tranquillité et de l'ordre aux habitants eux-mêmes, [pour] leur inspirer la sécurité et éviter tout malentendu et toute altercation entre les habitants et la force armée ${ }^{46}$ ". Bref, cette ellipse remet en cause le mouvement de fond de prise en charge de la sûreté individuelle des communautés aux agents de l'État ${ }^{47}$. Le retour au calme ne suffit pas : il s'agit de rétablir l'ordre public, c'est-à-dire l'ordre assuré par la force publique :

"L'armement spontané d'une partie de la population sans l'intervention des magistrats peut, dans un moment de crise où l'organisation politique ébranlée laisse les citoyens dans l'incertitude de leurs devoirs et de leur destinée, être excusée par les circonstances ou les motifs qui y ont donné lieu : un pareil mouvement serait un véritable et funeste délit sous un gouvernement régulier, unanimement reconnu, chéri et respecté des peuples et qui n'a besoin pour assurer l'exercice d'une autorité légitime que de l'action des lois ${ }^{48}$."

C'est dire que l'enjeu du port d'arme ne se réduit pas à celui du contrôle public de la sûreté individuelle : il interroge aussi le lien politique entre les administrés et le pouvoir parce qu'il favorise des formes d'expression populaire exclues du cadre censitaire qui doit s'instaurer. Les débats ont en effet débordé du cercle des élites; Barante évoquait ainsi des " esprits ayant été entraînés hors de leur sphère ordinaire ${ }^{49}$ ". C'est là un élément structurant de l'appréciation de l'esprit public, à savoir l'assignation pour chaque groupe social d'un ensemble de préoccupations ordinaires. Il y a signe ou risque de déstabilisation lorsque les cultivateurs, manipulés par le haut, sortent du champ de leurs intérêts matériels quotidiens.

Il importe donc de refouler les masses hors du jeu politique, sous peine de guerre civile parce qu'emportées par leurs passions, elles ne sauraient s'en tenir au débat d'opinion. Ce reflux passe par la re-sédentarisation des populations, avec l'idée sous-jacente qu'en prenant le fusil, qu'en s'enfonçant dans les bois et le bocage, elles ont abandonné leurs intérêts naturels et que par conséquent seul le retour aux travaux des champs les éloignera des conflits politiques. Le rétablissement du bon ordre exige que chacun

45. Le maire de Nantes au commissaire à la Police générale, 9 mai 1814, Arch. nat., $\mathrm{F}^{7} 9099$.

46. Le préfet de Maine-et-Loire au commissaire à la Police générale, 4 mai 1814, Arch. nat., $\mathrm{F}^{7} 9111$.

47. LignereuX, Aurélien, La France rébellionnaire. Les résistances à la gendarmerie (18001859), Rennes, Presses universitaires de Rennes, 2008, 365 p.

48. Les commissaires Ruty et d'Osmond au préfet de Maine-et-Loire, 23 mai 1814, Arch. dép. du Maine-et-Loire, 1 M 623.

49. Le préfet de la Vendée au commissaire à la Police générale, 30 avril 1814, Arch. nat., $\mathrm{F}^{7} 9250$. 
reprenne sa place. Le clergé est sollicité. Le gouvernement prend même le risque, pour accréditer ses agents, d'entériner l'autorité des chefs vendéens, en espérant qu'un tel relais ramène le calme (leur absence expliquerait le retour des troubles à Clisson, le $10 \mathrm{mai}^{50}$ ), tout en faisant rentrer ces derniers dans le rang et en ternissant leur prestige à l'issue d'un désenchantement tant leur nouveau rôle - faire payer les contributions - est ingrat.

Quant aux chefs secondaires, les administrateurs, qui ne voient en eux que des ambitieux, comptent les neutraliser par quelques places ou pensions. Ces récompenses particulières devraient permettre de faire l'économie d'une reconnaissance générale des services que les Vendéens estiment avoir rendu à la monarchie, non seulement pendant la Révolution mais encore dans la chute de Napoléon. Paris refuse cette dernière dette et entend pour le reste s'en tenir à l'estime morale, sans déroger à la charte octroyée. Une concession tels qu'une remise des impôts ou le rétablissement du statut de marche ne saurait être accordée sous la pression. Ce serait miner toute autorité et fissurer le socle même du Nouveau Régime sur lequel repose la monarchie restaurée. Barante tourne en dérision l'espoir de "privilèges particuliers à cause de leur dévouement à la cause royale ${ }^{51}$ ".

Le roi entend être celui de tous les Français et la même loi doit s'appliquer partout. Cette indifférenciation entre les Français et les Vendéens, et parmi ceux-ci entre les bleus et les blancs, poussent les plus résolus à miser sur un nouveau roi, porteur d'une autre conception de la monarchie. Dès avril 1814, Busche lançait cet avertissement : «L'autorité du Gouvernement, des fonctionnaires est complètement méconnue. Celle du roi pourrait le devenir également. L'on a déjà entendu des paysans dire l'Empereur nous a contraint de désirer Louis XVIII, mais si celui-ci se montre trop exigeant, nous crierons bientôt vive Louis XIX ${ }^{52}$. " La Restauration met au jour l'écart entre la pratique monarchique et l'imaginaire de la royauté cultivée par les Vendéens. Gilbert des Voisins explicite le choc qu'a représenté la Restauration pour ces derniers, comme celui d'une révélation qu'il y a d'autres façons d'être royaliste et que le roi peut même préférer les ralliements de raison : "La France entière, dans l'opinion des gens du païs, est dans la Vendée; tout ce qui leur convient doit convenir à tout le royaume ${ }^{53}$. " Or c'est à la Vendée de s'aligner sur la France; elle doit vivre désormais au même rythme que le pays, ce qui suppose l'ouverture de routes, comme y invite ce commissaire du roi, dans la continuité du discours tenu sous Napoléon.

50. Le préfet de la Loire-inférieure au commissaire à l'Intérieur, 11 mai 1814, Arch. nat., F 1cIII L-I, 11.

51. Le préfet des Deux-Sèvres au commissaire à l'Intérieur, 17 mai 1814, Arch. nat., $\mathrm{F}^{7} 9099$.

52. Le préfet des Deux-Sèvres au commissaire à la Police générale, 23 avril 1814, Arch. nat., $\mathrm{F}^{7} 9226$.

53. Le commissaire extraordinaire du roi au ministre de l'Intérieur, 25 juin 1814, Arch. nat., $F^{7} 7029$. 
Dans les premiers jours de mai 1814, des milliers de paysans vendéens, pris de peur, ont de nouveau saisi les armes. Battant le bocage pour y débusquer un ennemi imaginaire, né du traumatisme révolutionnaire et des angoisses présentes, ils ont ainsi entériné l'identité singulière de la Vendée militaire, rebelle aux prescriptions officielles ${ }^{54}$. Bien qu'éphémère, cette démonstration met à nu les antagonismes locaux et la fragilité du contrôle de l'administration. Celle-ci s'est efforcée de nommer - et de disqualifier - cette agitation en déclinant le champ lexical du soulèvement : " espèce d'insurrection ", "révolution ", " véritable rébellion contre l'autorité royale ". Cette terminologie séditieuse, que justifie en partie la forme d'un mouvement fort proche de juillet 1789, mais une Grande Peur dont la teneur a été irréversiblement infléchie par le choc de 1793-1794, a finalement été désavouée : " Donner à cette effervescence le nom de tentatives criminelles, y voir un germe de révolte, de guerre, c'est aller trop loin ${ }^{55}$. " Dans les circonstances du printemps 1814, la sagesse politique compte autant que la justesse sémantique. Deux expressions conjuguent toutefois ces qualités. D’une part, celle de levée en masse léguée par la Révolution et qu'emploient aussi bien les préfets Busche et Barante. D'autre part, celle de levée de boucliers, expression éloquente dont se sert par exemple le maire de Nantes pour caractériser cette protestation armée mais défensive. La démonstration s'en est tenue là, le pouvoir s'étant résigné à éviter l'épreuve de force. Face à cette manifestation d'une identité politique soudée par les épreuves, la peur et l'espoir, la réplique s'est orientée vers une entreprise de désarmement et de dépolitisation, dont les effets se font sentir dès mars 1815, avec la relative passivité des Vendéens face au retour de Napoléon..., passivité qu'entretiennent dans une certaine mesure les autorités royalistes, redoutant plus que tout et jusqu'au dernier moment une prise d'armes incontrôlée.

54. Sur cette "insoumission vendéenne ", Martin, Jean-Clément, La Vendée de la mémoire, 1800-1980, Paris, Éd. du Seuil, 1989, p. 37-64.

55. Le directeur général de la police au préfet des Deux-Sèvres, 11 juin 1814, Arch. nat., $F^{7} 9226$. 


\section{RÉSUMÉ}

Les révolutionnaires ont été confrontés à "l'inexplicable Vendée ", le soulèvement de tout un peuple contre une révolution menée au nom du peuple. Vingt ans plus tard, les certitudes de la Première Restauration sont momentanément ébranlées par une incompréhension du même ordre : la levée de boucliers de la "fidèle Vendée " contre les autorités reconnues par le roi légitime. Un mois après l'abdication de Napoléon, les communes de la rive gauche de la Loire prennent en effet les armes à l'appel du tocsin; du 3 au 5 mai 1814, plusieurs dizaines de milliers d'hommes tiennent les campagnes en opposition frontale aux autorités légales. Qui étaient ces insurgés? Comment et pourquoi une telle mobilisation a-t-elle eu lieu? Voilà qui invite à une approche totale de cet événement méconnu et pourtant si révélateur du traumatisme régional, dans son déroulement factuel ainsi que dans ses dimensions politiques, sociales et culturelles.

\section{ABSTRACT}

The members of the Convention were confronted by "the inexplicable Vendée", a people's uprising against what claimed to be a people's revolution. Twenty years later, a similar incomprehension disturbs the certainties of the First Restoration: the revolt of the "faithful Vendée" against the authorities recognized by the legitimate king. A month after Napoleon's abdication, the inhabitants of the south bank of the Loire took up arms to the call of the tocsin; from the $3^{\text {rd }}$ to the $5^{\text {th }}$ of May 1814, thousands of armed men held the country in a frontal opposition to the legal authorities. Who were these rebels? Why and how did such a mobilization occur? This article proposes a global approach, factual and political, social and cultural, to an event which reveals the regional revolutionary trauma, but is nevertheless largely ignored by historians. 